\title{
Die Umdeutung mythischer Zweideutigkeit
}

Dialektik im Stillstand und Opfer der Aufklärung bei Benjamin und Adorno

La réinterprétation de l'ambiguité mythique. Dialectique à l'arrêt et sacrifices de rationalité chez Benjamin et Adorno

The Reinterpretation of Mythical Equivocality. Dialectics at a Standstill and

Sacrifices of Enlightenment in Benjamin and Adorno

\section{Tilman Reitz}

\section{OpenEdition \\ Journals}

Édition électronique

URL : http://journals.openedition.org/ceg/4312

DOI : $10.4000 /$ ceg.4312

ISSN : 2605-8359

\section{Éditeur}

Presses Universitaires de Provence

Édition imprimée

Date de publication : 2 mai 2019

Pagination : 49-62

ISBN : 979-10-320-0214-8

ISSN : 0751-4239

\section{Référence électronique}

Tilman Reitz, „Die Umdeutung mythischer Zweideutigkeit", Cahiers d'Études Germaniques [Online], 76। 2019, Online erschienen am: 02 November 2020, abgerufen am 24 Januar 2021. URL: http:// journals.openedition.org/ceg/4312 ; DOI: https://doi.org/10.4000/ceg.4312 


\section{Die Umdeutung mythischer Zweideutigkeit Dialektik im Stillstand und Opfer der Aufklärung bei Benjamin und Adorno}

Tilman REITZ

Friedrich-Schiller-Universität Jena

Ein Buch, das den Kapitalismus als Machtordnung erklärt, nutzt die kulturwissenschaftlich beliebte, verschiedenen Indianerstämmen zugeschriebene Auffassung, dass vor uns nicht die Zukunft, sondern das Gewesene liegt. Derartig bewegen sich, so die These, auch Firmen und Anlegerinnen im Zeitverlauf. Zwar gälten „capitalists“ allgemein als „forward looking“, weil von der Zukunft besessen; auch ihnen ist diese Zukunft jedoch unbekannt, und Orientierung können sie nur mit Blick auf bereits erzielte Renditen gewinnen, sodass sie gleichsam rückwärts gehen: „Da unsere Augen nur sehen können, was vor uns, und blind sind für das, was hinter uns liegt, ist es sinnvoller zu sagen, dass die Kapitalisten die Zukunft hinter sich liegen haben“. ${ }^{1}$ Während sie immer weiter Zahlungskraft und -verpflichtungen aufhäufen, schauen sie ständig auf das Geschehene.

Walter Benjamin hat in zwei Texten, zwischen deren Abfassung gut zwanzig Jahre liegen, Bilder für Zeitlichkeit im Kapitalismus gefunden, die dieser Darstellung auffällig ähneln. Im ersten Text, einem wohl um 1919 zu datierenden Fragment, stellt er den Kapitalismus als „Religion“ dar, die ohne theologische Lehre auskommt, aber Alltag und Beziehungen der Menschen mit einem unausgesetzt zu praktizierenden Kult strukturiert. Zu diesem Kult zählt wesentlich die Anhäufung von Schulden, die nun das beherrschende Medium intersubjektiver Verbindlichkeiten bilden:

Der Kapitalismus ist vermutlich der erste Fall eines nicht entsühnenden, sondern verschuldenden Kultus. Ein ungeheures Schuldbewusstsein[,] das sich nicht zu entsühnen weiß, greift zum Kultus, um in ihm diese Schuld [...] universal zu machen, dem Bewusstsein sie einzuhämmern und endlich und vor allem den Gott selbst in die Schuld einzubegreifen[,] um endlich ihn selbst an der Entsühnung zu interessieren. ${ }^{2}$

1. ,[S]ince our eyes can only see what lies ahead and are blind to what lies behind, it makes more sense to say that capitalists have the future behind them". Jonathan Nitzan, Shimshon Bichler, Capital as Power, New York, Routledge 2009, S. 187.

2. Walter Benjamin, „Kapitalismus als Religion“, in Gesammelte Schriften, hrsg. v. Rolf Tiedemann u. Hermann Schweppenhäuser, Frankfurt a. M. 1980, Suhrkamp Verlag, Bd. VI, S. 100-103, hier $100 f$. 
Der zweite Text, die wohl in Benjamins letztem Lebensjahr niedergeschriebenen Thesen „Über den Begriff der Geschichte“, deuten auf berühmt gewordene Weise Paul Klees Zeichnung „Angelus Novus“ als Sinnbild dafür, dass auch die Befreiungshoffnungen einer ihrer Zukunft nicht mächtigen Menschheit nur darauf zielen können, bereits erlittenes Unrecht zu korrigieren.

Der Engel der Geschichte [...] hat das Antlitz der Vergangenheit zugewendet. Wo eine Kette von Begebenheiten vor uns erscheint, da sieht er eine einzige Katastrophe, die unablässig Trümmer auf Trümmer häuft und sie ihm vor die Füße schleudert. ${ }^{3}$

Benjamins Schilderungen (mangels Fortgang bzw. angesichts des ausstehenden Endes ist hier kaum von ,Erzählungen“ zu sprechen) scheinen selbst mythische Qualität zu haben; im ersten, früheren Fall werden vielleicht sogar christliche Inhalte remythisiert. Fragt man, weshalb sich dieser Eindruck einstellt, treten Umrisse dessen hervor, was im Folgenden bei Benjamin sowie Adorno und Horkheimer als (Arbeit am) Mythos untersucht werden soll. Das erste Merkmal betrifft den kulturellen Status der Texte bzw. Motive: Man kennt sie inzwischen vom Hörensagen. Fortschrittskritik kann sich problemlos auf den Engel der Geschichte, Kapitalismuskritik auf Kapitalismus als Religion beziehen. Diese Rolle konnten Benjamins Schilderungen erlangen, weil sie zweitens das semantische Angebot machen, in immer wieder neu lesbarer Weise über unser Schicksal zu berichten. Das Bild vom Trümmerhaufen kann man auf die Krisendynamik kapitalistischer Akkumulation oder auf politische Niederlagen der Arbeiterbewegung beziehen, aber auch auf die spezifischen Prozesse, die zu Auschwitz geführt haben; im Kapitalismus als Religion kann man sowohl den Zug zur universellen Verschuldung als auch Aspekte der Marktgläubigkeit betonen. In signifikanter Weise mythisch ist drittens auch der Inhalt der Texte selbst. Sie betreffen tatsächlich eine Art Schicksal, indem sie offenbar unausweichliche Prozesse schildern, für die eine Blindheit der Handelnden konstitutiv ist. Das macht sie geeignet, Situationen und Zustände zu erfassen, die so niemand gewollt haben kann, und bringt sie in Spannung zu den Ansprüchen bewusster Weltgestaltung, die in der Moderne bekanntlich stark angewachsen sind.

Diese Spannung ist uns seit Horkheimer und Adorno als Dialektik der Aufklärung vertraut. Ich will im Folgenden einen weiteren Beitrag zur Genealogie dieser Figur leisten, weil sie gerade angesichts der im Frankfurter Umkreis geleisteten Arbeit am Mythos keineswegs direkt einleuchtet. Während besonders Benjamin in der Weimarer Zeit einen durchgängig kritischen Mythosbegriff entwickelt hatte, den man ohne Weiteres auf die Mythisierungen der Nazis hätte anwenden können, gehen er und (expliziter) Adorno im Exil dazu über, auch positive Seiten des Mythos herauszustellen - von der Naturnähe der Mimesis bis zur ,Zweideutigkeit‘ oder rückblickend erkennbaren Offenheit politisch-geschichtlicher Entscheidungssituationen. Meine Frage wird sein, inwiefern sich diese Wende aus der eigenen Situation der beiden Exilanten heraus erklären bzw. als strategische Neubesetzung verstehen lässt. Als Kontrastfall wird mir Ernst Cassirer dienen,

3. Walter Benjamin, „Über den Begriff der Geschichte“, in Gesammelte Schriften, Bd. I.2, S. 691-704, hier 697. 
dessen Mythosbegriff sich genau umgekehrt und damit prima facie verständlicher verschiebt: Von der zunächst eher aufgeschlossenen Einschätzung des Mythos als Erkenntnisform geht er in der Tat dazu über, politische Mythologie als solche und besonders ihre nationalsozialistische Form anzugreifen. Weshalb sich Benjamin und Adorno anders entscheiden, lässt sich am besten ihren Entwürfen entnehmen, zumal Adornos (1943 abgeschlossenem, 1998 posthum publiziertem) Manuskript zum Odysseekapitel der Dialektik der Aufklärung ${ }^{4}$ und Benjamins Exposé zum Passagenwerk (das in deutscher Fassung 1935, in französischer 1939 abgefasst wurde). ${ }^{5}$ Die Diskussion dieser Texte wird unterschiedliche Strategien der MythosAufwertung hervortreten lassen, die jedoch beide erkennbare Gegenentwürfe zur Nutzung des Mythos im nationalsozialistischen Deutschland darstellen. Die Struktur des Beitrags folgt diesen Erwägungen: Ich werde erstens Benjamins kritischen Mythosbegriff rekonstruieren und dessen (nicht nur politische) Anschlussfähigkeit mit Cassirer veranschaulichen, zweitens die Dialektisierung des Mythos bei Horkheimer und Adorno kurz rekapitulieren und etwas länger herleiten, um drittens Benjamins Aufwertung von ,Zweideutigkeit' als Alternative darzustellen - um viertens abschließend zu erläutern, inwiefern hier zwei pointierte Versionen des Mythos im Exil vorliegen.

\section{Mythos als zweideutiger Zwang: Grundlegung beim frühen Benjamin}

Für den frühen Benjamin ist Mythos ein zentraler Ablehnungsbegriff. Er bezeichnet zunächst bloße, vorrechtliche Machtverhältnisse, darauf aufbauend ein Schicksal, dem man naturhaft ausgeliefert ist, sowie schließlich eine intransparente, ,zweideutige' Ordnung von Schuld und Sühne. Dieses mehrschichtige Muster verfolgt Benjamin von den alten Göttergeschichten bis in die moderne Rechtspflege. Es fußt auf mehr oder minder klar stilisierten Konstruktionen eines vorrechtlichen Zustands: „Die mythische Gewalt in ihrer urbildlichen Form ist bloße Manifestation der Götter. Nicht Mittel ihrer Zwecke, kaum Manifestation ihres Willens, am ersten

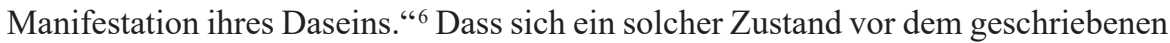
oder überhaupt bekannten Recht als ,zweideutig' erweist, sobald (trotzdem) Fehlverhalten bestraft wird, folgt unmittelbar:

Gesetzte und umschriebene Grenzen bleiben, wenigstens in Urzeiten, ungeschriebene Gesetze. Der Mensch kann sie ahnungslos überschreiten und so der Sühne verfallen. [...] Aber so unglücklich sie den Ahnungslosen treffen mag, ihr Eintritt ist im Sinne des Rechts nicht Zufall, sondern Schicksal, das sich hier [...] in seiner planvollen Zweideutigkeit darstellt. ${ }^{7}$

4. Theodor W. Adorno, „Geschichtsphilosophischer Exkurs zur Odyssee. Frühe Fassung von Odysseus oder Mythos und Aufklärung“, Frankfurter Adorno Blätter V, 1998, S. 37-88; zur Datierung (und zur Autorschaft Adornos) vgl. die editorische Notiz von Rolf Tiedemann, S. 37.

5. Vgl. den editorischen Bericht Rolf Tiedemanns in Walter Benjamin, Gesammelte Schriften, Bd. V.2, S. 1067-1080, hier 1074 .

6. Benjamin: „Zur Kritik der Gewalt“ (1921), in Gesammelte Schriften, Bd. II.1, S. 179-203, hier 197.

7. Ibid., S. 199. 
Zweideutig ist wohl vor allem die Strafe, die zwischen normativ motivierter Sanktion und natürlich-notwendiger Folge des Fehlverhaltens changiert. Für die grundlegende Kritik des Rechts, die Benjamin im Kontext anstrebt, ist entscheidend, dass auch der moderne Staat nie ganz von der, rechtssetzenden Gewalt ${ }^{6}$ und der Blindheit des Schicksals loskommt, also einer ,mythischen Zweideutigkeit der Gesetze` verhaftet bleibt. $^{8}$

Wie immer man diese Rechtskritik einschätzt, ${ }^{9}$ der entsprechende Mythosbegriff ist prägnant und gut erweiterbar. In seinem Essay zu Goethes Wahlverwandtschaften wendet ihn Benjamin u.a. erkenntnistheoretisch - und lehnt auch hierbei den Mythos strikt ab:

Es gibt keine Wahrheit, denn es gibt keine Eindeutigkeit und also nicht einmal Irrtum im Mythos. [...] Da es aber ebenso wenig Wahrheit über ihn geben kann [...], so gibt es, was den Geist des Mythos angeht, einzig und allein eine Erkenntnis [...-] nämlich die Erkenntnis von seiner vernichtenden Indifferenz gegen die Wahrheit. ${ }^{10}$

Die letzte Schlussfolgerung wirft Fragen für die geisteswissenschaftliche Forschung auf, die in gewisser Weise von der Ausdeutung der in Mythen chiffrierten Grunderfahrungen lebt. Bis auf die streitbare Zuspitzung dieses Punkts bewegt sich Benjamins Mythosverständnis aber nah an verbreiteten Annahmen. Ein oft genutzter Schlüssel für die in der Rechtskritik wie im Wahlverwandtschaften-Essay prominente ,Zweideutigkeit‘ liegt in der Idee, dass mythisches Denken keine Grenze zwischen kausalen Wirkungsketten und semantischen Verhältnissen, ${ }^{11}$ z.B. zwischen Vorzeichen und Ursache, Handlungsfolgen und Strafe zieht. So sieht es zeitgleich mit Benjamin namentlich Ernst Cassirer:

Wo wir ein Verhältnis der bloßen ,Repräsentation“ sehen, da besteht für den Mythos, sofern er von seiner Grund- und Urform noch nicht abgewichen und von seiner Ursprünglichkeit noch nicht abgefallen ist, vielmehr ein Verhältnis realer Identität. Das ,Bild‘ stellt die ,Sache“ nicht dar - es ist die Sache; es vertritt sie nicht nur, sondern wirkt gleich ihr, so dass es sie in ihrer unmittelbaren Gegenwart ersetzt. ${ }^{12}$

Cassirer erkennt hier noch nicht die Möglichkeit, die Ungeschiedenheit von symbolischer und kausaler Ordnung nicht nur zur Analyse eines archaischen Denkens, sondern auch zur Kritik der Gegenwart zu nutzen. Eben diese Möglichkeit

8. Es ist „dieselbe mythische Zweideutigkeit der Gesetze, von der Anatole France satirisch spricht, wenn er sagt: Sie verbietet es Reichen wie Armen gleichermaßen, unter Brückenbogen zu nächtigen“. Ibid., S. 198.

9. In letzter Zeit wurde oft an sie angeschlossen; durch die aporetische Grundidee, dass die Rechtssetzung selbst nie rechtskonform, sondern eben immer gewaltsam ist, empfiehlt sie sich Autoren wie Derrida (Force de loi. Le 'Fondement mystique de l'autorité', Paris 1990, Galilée), Giorgio Agamben (Ausnahmezustand. Homo Sacer, II.2, Frankfurt a. M., 2004, Suhrkamp) oder Judith Butler.

10. Benjamin, „Goethes Wahlverwandtschaften“, in Gesammelte Schriften, Bd. I.1, S. 123-201, hier 162.

11. So steht es fast wörtlich bei Lévi-Strauss: „Das wilde Denken ist [...] auf dieselbe Weise logisch, wie es unser Denken ist, aber nur“ als „Erkenntnis einer Welt, der es zugleich physische und semantische Eigenschaften zuschreibt.“ Claude Lévi-Strauss, Das wilde Denken (La pensée sauvage, 1962), Frankfurt a. M. 1968, Suhrkamp, S. 308.

12. Ernst Cassirer, Philosophie der symbolischen Formen, Bd. 2, Hamburg, Meiner, 2010, S. 47. 
entdeckt er jedoch in seiner späten, im US-amerikanischen Exil geschriebenen Kritik politischer Remythisierung.

[D] as magische Wort [...] benennt nicht Dinge oder Beziehungen zwischen Dingen; es versucht, Wirkungen hervorzubringen. [...] Seltsam genug, alles dies kehrt in unserer modernen Welt wieder. Wenn wir unsere modernen politischen Mythen [...] studieren, so finden wir in ihnen zu unserer großen Überraschung [...] eine Umformung der menschlichen Sprache. Das magische Wort gewinnt Oberhand über das semantische Wort. ${ }^{13}$

Zur gleichen Zeit, in der Cassirer derart den Kampf zwischen Mythos und Logos neu aufzunehmen empfiehlt, sind Adorno und Horkheimer allerdings schon dabei, dem Mythos Rettungspotenzial abzugewinnen, und Benjamin rehabilitiert die früher scharf verurteilte Zweideutigkeit. Im Folgenden will ich nachvollziehen, was sie jeweils dazu veranlasst.

\section{Mythische Opfer: Teilrehabilitierungen in der „Dialektik der Aufklärung“"}

Adorno hat bereits in seiner Antrittsvorlesung von 1931 mit einem innovativen (und nicht nur kritischen) Mythosverständnis experimentiert. ${ }^{14}$ In der Dialektik der Aufklärung übernehmen Horkheimer und er zunächst Benjamins Auffassung des Mythos als kulturell fortgesetztem Naturzwang und Schicksalsverfallenheit; sie ergänzen (ein anderes Hauptmotiv Benjamins aufnehmend), dass der Mythos damit vor allem für zyklische Zeit oder ewige Wiederholung steht:

Alle Geburt wird mit dem Tod bezahlt, jedes Glück mit Unglück. Menschen und Götter mögen versuchen, in ihrer Frist die Lose nach anderen Maßen zu verteilen als der blinde Gang des Schicksals, am Ende triumphiert das Dasein über sie. ${ }^{15}$

Sie sehen sogar wie Cassirer das Problem mythischer Sprache („Das Wort soll unmittelbar Macht haben über die Sache“ ${ }^{16}$ ) und erkennen an, dass Rationalität ein Ausweg aus dem Schuldkreislauf sein könnte:

Im Mythos gilt jedes Moment des Kreislaufs das voraufgehende ab und hilft damit, den Schuldzusammenhang als Gesetz zu installieren. Dem tritt Odysseus entgegen. Das Selbst repräsentiert rationale Allgemeinheit wider die Unausweichlichkeit des Schicksals. ${ }^{17}$

Horkheimer und Adorno setzen jedoch ihren bekannten eigenen Akzent, indem sie dem Mythos Aufklärungsziele zuschreiben und die mythenkritische Aufklärung

13. Cassirer, Vom Mythus des Staates, Zürich 1949, Artemis Verlag, S. $368 f$.

14. Vgl. Rolf Tiedemann, „, Gegenwärtige Vorwelt‘. Zu Adornos Begriff des Mythischen“, Frankfurter Adorno Blätter V, 1998, S. 9-36.

15. Max Horkheimer, Theodor W. Adorno, Dialektik der Aufklärung. Philosophische Fragmente, Frankfurt a. M. 1988, Fischer Verlag, S. 22.

16. Ibid., S. 67.

17. Ibid., S. 66. 
selbst als mythisch, weil naturzwanghaft begreifen. ${ }^{18}$ Die erste Wendung ist gut nachvollziehbar: „Der Mythos wollte berichten, nennen, den Ursprung sagen: damit aber darstellen, festhalten, erklären."19 Die Figur des Naturzwangs wird demgegenüber mit zahlreichen Zusatzgedanken umspielt, etwa mit einer Reflexion über Aussageverbote: „Aufklärung ist radikal gewordene mythische Angst. Die reine Immanenz des Positivismus, ihr letztes Produkt, ist nichts anderes als ein gleichsam universal gewordenes Tabu. " ${ }^{20} \mathrm{Zu}$ den erklärend-erweiternden Gedanken zählt nicht zuletzt, dass Mythoskritik selbst in ein kritisches Licht rückt:

Das mythische Grauen der Aufklärung gilt dem Mythos. Sie gewahrt ihn nicht bloß in unaufgehellten Begriffen und Worten, wie die semantische Sprachkritik wähnt, sondern in jeglicher menschlichen Äußerung, wofern sie keine Stelle im Zweckzusammenhang [der] Selbsterhaltung hat. ${ }^{21}$

Der Mythos wird damit umgekehrt zum Hoffnungsträger. In Adornos Lektüre der Odyssee $^{22}$ steht er tendenziell für die Gesamtheit dessen, was im Fortschrittsprozess geopfert wurde. Der Protagonist Odysseus behauptet bzw. gewinnt sein rationales Selbst für Adorno wesentlich durch eine „Introversion des Opfers“; ${ }^{23}$ was er durch Überlistung der Götter überwindet und hinter sich lässt, sind sämtlich mythische Glücksversprechen. Die Beispiele reichen von der „Idylle der Lotophagen“ mit ihrem „wie sehr auch selber befangenen Schein der Erlösung“24 über die zur „Auflösung“ treibende Hingabe bei Kirke bis zum „Drang, Vergangenes zu erretten, anstatt es als Stoff des Fortschritts zu benützen“, bei den Sirenen: „Indem sie jüngst Vergangenes unmittelbar beschwören, bedrohen sie mit dem unwiderstehlichen Versprechen von Lust, als welches ihr Gesang vernommen wird, die patriarchale Ordnung“" ${ }^{25}$

Eine Humanität, die den Mythos bloß bekämpft, verliert also immer Entscheidendes. Diese Rekonstruktion ist als Lesemuster wohl der schlichten Gegenüberstellung von Mythos und Rationalität bei Cassirer überlegen. Bei ihm verspricht eine Gestalt wie Kirke nur Regression:

Wir haben erfahren, dass der moderne Mensch trotz seiner Ruhelosigkeit, oder vielleicht gerade wegen seiner Ruhelosigkeit, den Stand des unzivilisierten Lebens nicht wirklich überwunden hat. [...] Von allen traurigen Erfahrungen der letzten zwölf Jahre ist diese vielleicht die furchtbarste. Sie mag mit der des Odysseus auf der Insel der Kirke verglichen werden. [...] Kirke hatte die Gefährten des Odysseus in verschiedene Tiergestalten verwandelt. [...] Sie haben aufgehört, freie und persönlich handelnde Menschen zu sein. ${ }^{26}$

18. Die verknappte Formel lautet: ,[S]chon der Mythos ist Aufklärung, und: Aufklärung schlägt in Mythologie zurück“" (Ibid., S. 6).

19. Ibid., S. 14.

20. Ibid., S. 22

21. Ibid., S. 35 .

22. So muss man es nach der Publikation von Adornos Manuskript (siehe Fn.3) wohl formulieren. Wenngleich die Dialektik der Aufklärung in Ko-Autorschaft mit Horkheimer publiziert (und auch unter Mitwirkung von Gretel Adorno verfasst) wurde, wurden alle Hauptargumente des Odyssee-Kapitals zunächst von Adorno niedergeschrieben.

23. Ibid., S. 62.

24. Ibid., S. 77.

25. Ibid., S. 39.

26. Cassirer, Mythus des Staates, S. 373. 
Adorno teilt zwar die Einschätzung, dass Kirke die Autonomie der ankommenden Männer bedroht, ${ }^{27}$ wendet sich aber zugleich gegen die patriarchalische Lösung, dass der Mann seinen Trieb aufschiebt und als Belohnung Herrschaft über die Frau gewinnt. Erst so wird die alte Geschlechterideologie kritisierbar, mit der im 20. Jahrhundert gerade der Nationalsozialismus gearbeitet hat.

Aus dem Bezugskontext faschistischer Remythisierung lässt sich auch insgesamt die Arbeit am Mythos begreifen, die Adorno an der Odyssee leistet bzw. in der Interpretation der bei Homer geleisteten Mythenverarbeitung erneut aufnimmt. Er führt hier, wie sein posthum publiziertes Manuskript deutlich macht, einen ZweiFronten-Kampf mit den vorherrschenden deutschen Deutungslinien, der seine zeitgleich entwickelte Dialektik der Naturbeherrschung politisch lesbar macht. Einerseits kritisiert Adorno die „humanistische Tradition“, die sich um jeden Preis von unklaren, nicht legitimatorisch nutzbaren Ursprüngen lösen will:

Der herrische Ton ihrer späten Meister, Kirchhof oder gar Wilamowitz, der von der Prähistorie so wenig etwas wissen wollte wie von Nietzsche, entspringt bereits der Verzweiflung daran, das antike Erbe als Wahrheit festhalten zu wollen, das auseinanderfiel in krude und selbst als solche bedenkliche Faktizität und in Kulturphilosophie. ${ }^{28}$

Noch sichtlich stärker grenzt er sich jedoch von der Deutungslinie ab, die den echten, kraft- und gewaltvollen Mythos von seiner mäßigenden, zivilisierenden Bearbeitung durch Homer befreien will. Adorno bestreitet nicht die sachliche Triftigkeit dieses Ansatzes; er exzerpiert dazu passende Thesen wie die, dass bei „Homer durch zivilisatorische Zensur Folterszenen getilgt“ sind. ${ }^{29}$ Wenn man aber den Impuls gegen Folter nicht einfach als Verfälschung lesen will, ist Vorsicht gegenüber den Anhängern des ursprünglichen Mythos geboten. Das gilt zumal für den abtrünnigen George-Schüler Rudolf Borchardt. „Borchardt hat“ Adorno zufolge „den antimythologischen, aufgeklärten Charakter Homers erkannt", ${ }^{30}$ doch zugleich zeige er, wie ,die Hüter des Echten ihrer Verschworenheit mit der Barbarei innewerden“. ${ }^{31}$ Im Kontext ordnet Adorno Borchardts Schriften als „am Rande der deutschen Großindustrie gepflegte Esoterik" ein und schreibt ihnen auch sachlich den Standpunkt des Industriekapitals zu: „Homer, die kolonialgriechische Erzählung, wird zu der von Seefahrern und Händlern gestempelt, die dem Hofpoeten der Fabrikherren verächtlich sind.“"32 Folgerichtig mündet „die ästhetische Kritik des Klassizismus politisch in Apologie der heraufziehenden Diktaturen“. ${ }^{33}$

27. „Die Hetäre gewährt Glück und zerstört die Autonomie des Beglückten“ (Horkheimer/ Adorno, Dialektik der Aufklärung, S. 78).

28. Adorno, „Geschichtsphilosophischer Exkurs zur Odyssee“, S. 38.

29. Ibid., S. 88, sowie Horkheimer, Adorno, Dialektik der Aufklärung, S. 87: Adorno bezieht sich hier auf Gilbert Murray, seinen Gewährsmann für sachlich-moderne Homer-Philologie.

30. Ibid., S. 43 .

31. Ibid., S. 39.

32. Ibid. (zu den „Esoterikern der deutschen Schwerindustrie“ zählt Borchardt auch in Horkheimer, Adorno, Dialektik der Aufklärung, S. 52).

33. Ibid., S. 40. 
Diese Urteile sind angesichts der zitierten Borchardt-Stellen überwiegend nachvollziehbar. ${ }^{34}$ Sie verstärken allerdings die Asymmetrie, die bereits in der schematischen Gegenüberstellung von Mythos und Aufklärung spürbar war: Wenn das ursprünglich Mythische und die Begeisterung dafür so düster aussehen, scheint es wenig Gründe zu geben, nicht einfach aufklärerisch an seiner Tilgung $\mathrm{zu}$ arbeiten. Besonders deutlich wird das an einer ins Buch übernommenen Stelle des Manuskripts, die ähnlich wie später Rancière vom egalisierenden Geist der literarischen Form handelt:

Die homerische Rede schafft Allgemeinheit der Sprache, wenn sie sie nicht bereits voraussetzt; sie löst die hierarchische Ordnung der Gesellschaft durch die exoterische Gestalt ihrer Darstellung auf, selbst und gerade, wo sie jene verherrlicht; vom Zorn des Achill und der Irrfahrt des Odysseus Singen [sic] ist bereits sehnsüchtige Stilisierung dessen, was sich nicht mehr singen lässt. ${ }^{35}$

Während der erste Teil der Ausführung einleuchtet, projiziert der zweite einige Romantik in Gesänge, die vielleicht weniger mit Sehnsucht aufgeladen als selbst höfisch stilisiert waren. In jedem Fall leuchtet weder bei Adorno noch bei Borchardt ein, weshalb das Ursprüngliche und Poetische das Hierarchische und Volksferne gewesen sein soll. Deutlich ist hingegen, wie Adorno strategisch vorgeht: Er will die Stärke des Gegners aufnehmen. Statt dem „Talmi-Mythos der Faschisten“ ${ }^{\text {“36 }}$ bloß rationale Belehrung entgegenzusetzen, bemüht er sich um ein umfassenderes und freies Verständnis dessen, was im mythischen Stoff und seinen diversen Bearbeitungen thematisch ist.

Es bleibt die Frage, welche Alternativen Odysseus bzw. die Menschheit gehabt hätte. Wäre es besser gewesen, keine Pfade durchs Nebeneinander der Kulturen und Notlagen zu bahnen, die vor der Selbstbehauptung der Seefahrer, Krieger und Techniker anzunehmen sind? Sollte man doch den Versuch fortsetzen, vor der patriarchalischen Ordnung so etwas wie ein humaneres Mutterrecht zu entdecken? Adorno und Horkheimer verhandeln keine Alternativen dieser Art, weil sie alle mythischen Opfer in eine nun negativ (bzw. ,dialektisch') begriffene, aber nicht weniger lineare Fortschrittsgeschichte einordnen, statt wirkliche Entscheidungssituationen zu rekonstruieren. Je näher man die genauer bekannte Geschichte nachhomerischer Epochen betrachtet, desto fatalistischer sieht diese Finalisierung aus; sie gesteht noch nicht einmal zu, dass sich Gesellschaften an unterschiedlichen Orten auf verschiedene Pfade begeben.

34. Das gilt nicht für die Situierung Borchardts im Hofstaat der Industriebarone, die Adorno nur nahelegt, umso mehr aber für die politische Haltung, die er ihm zuschreibt. Aus Borchardts Buch Pindarische Gedichte von 1929 zitiert er: „,[D]er Sieg der Demokratie ist hier [zur Zeit der homerischen Epen] wie in Mittelalter und Neuzeit das Ende der Poesie. Was übrig bleibt, hier wie dort, ist Literatur, die der neuen Gesellschaft das Bild ihrer Misere liefert, und Romantik, die das der alten rekonstruiert, bis die Geschichte den Volkskehricht in die Sklavenecken neuer Herren räumt.“ (Zit. Ibid., S. 40)

35. Ibid., S. 40. Der Satz Adornos leitet im Manuskript die eben zitierte Ausführung Borchardts ein; dass letztere im Buch wegfällt, verschleiert sowohl die Quelle als auch den reaktionären Akzent des Gedankens.

36. Horkheimer, Adorno, Dialektik der Aufklärung, S. 19. 
Daher leuchtet es ein, dass Benjamin, der mindestens ebenso sehr an der Kraft der Gegenseite teilzuhaben versucht wie Adorno, eine andere Argumentationsstrategie wählt. Er rehabilitiert nicht den Mythos, sondern die Zweideutigkeit, die er nun neu als Möglichkeit auffasst, anders fortzufahren. Eben dies unterscheidet ja menschliche Zeichenordnungen von bloß natürlichen Kausalverkettungen.

\section{Zweideutigkeit in der Geschichte: Die Alternative(n) des Passagen-Exposés}

Benjamins Kennzeichnung des Mythos geht gleichsam abgekoppelt vom Bezugsbegriff in sein Ende der 1920er Jahre begonnenes, im Pariser Exil dann extensiv verfolgtes Hauptwerk ein. Alle Motive, die er in seinem Passagenwerk analysieren will, werden im Exposé Paris, die Hauptstadt des XIX. Jahrhunderts als zweideutig eingeführt. In den Weltausstellungen, die das „Universum der Waren“ präsentieren, erreicht die „Phantasmagorie der kapitalistischen Kultur [...] ihre strahlendste Entfaltung“"37 - doch zugleich können sich hier Delegierte der Arbeiterschaft versammeln, die den ästhetisch zugänglichen Warenreichtum auch praktisch zu besitzen beanspruchen. Im „Interieur" findet der „Privatmann“ sein „Etui“, das ihn scheinhaft von der Welt abschließt - doch zugleich „träumt“ er sich hier als „Sammler [...] nicht nur in eine ferne und vergangene Welt, sondern zugleich in eine bessere“, in der sogar „die Dinge von der Fron frei sind, nützlich zu sein". ${ }^{38}$ In der Umgestaltung des urbanen Raums durch Haussmann geht es Benjamin zufolge um „die Sicherung der Stadt gegen den Bürgerkrieg“639 - doch zugleich zeigt sie die Veränderbarkeit der städtischen Ordnung und erlaubt es, ,die Monumente der Bourgeoisie als Ruinen zu erkennen, noch ehe sie zerfallen sind". ${ }^{40}$ An der berühmten Figur des Flaneurs schließlich wird die "Zweideutigkeit" expliziert. Substanziell dürfte sie darin bestehen, dass ,,auf der Schwelle“ zur kommerziellen Funktionalisierung der Kunst ein ästhetisches Verhältnis zum Funktionsbündel Großstadt möglich wird. „Im Flaneur begibt sich die Intelligenz auf den Markt. Wie sie meint, um ihn anzusehen, und in Wahrheit doch schon, um einen Käufer zu finden. " ${ }^{41}$ Allgemein charakterisiert Benjamin seine ambivalenten Figuren an dieser Stelle als , dialektische Bilder' oder durch ,Dialektik im Stillstand': ,Zweideutigkeit ist die bildliche Erscheinung der Dialektik, das Gesetz der Dialektik im Stillstand. Dieser Stillstand ist Utopie und das dialektische Bild also Traumbild. "

Der Gehalt dieser Begriffsmontagen lässt sich klären, wenn man Benjamins Dialektik als Gegenentwurf zu derjenigen Hegels oder Adornos liest: Entscheidend ist nicht der Zug zur Auflösung oder Aufhebung der gespannten oder

37. Benjamin, „Paris, die Hauptstadt des XIX. Jahrhunderts“, in Gesammelte Schriften, Bd. V.1, S. 4559 , hier 51.

38. Ibid., S. $52 \mathrm{f}$

39. Ibid., S. 57.

40. Ibid., S. 59.

41. Ibid., S. 55.

42. Ibid. 
widersprüchlichen Konstellation, sondern ihr in der Selbstwahrnehmung wie in der engagierten Rekonstruktion offener Ausgang. Das hat den Vorteil, dass Alternativen sichtbar werden, aber den Nachteil, dass sie sich als bloße Wunsch- und Traumbilder oder (Effekte von) ,Phantasmagorien“ erweisen könnten. Benjamin expliziert diesen Gedanken ansatzweise in den klassisch-marxistischen Sätzen, in denen er den Fortgang der Geschichte mit der bildlichen Äußerung kollektiver Wünsche verbindet:

Der Form des neuen Produktionsmittels, die im Anfang noch von der des alten beherrscht wird,
entsprechen im Kollektivbewusstsein Bilder, in denen das Neue sich mit dem Alten durchdringt.
Diese Bilder sind Wunschbilder, und in ihnen sucht das Kollektiv die Unfertigkeit sowie die
Mängel der gesellschaftlichen Produktionsordnung sowohl aufzuheben wie zu verklären. ${ }^{43}$

Einiges hiervon wäre weiter zu diskutieren, etwa das Verhältnis zwischen der allgemeinen Struktur von Epochenbrüchen, der historisch einmaligen Entstehung der kapitalistischen Warenästhetik sowie den Klassen- und Staatsideologien des Zweiten Kaiserreichs. Im gegebenen Zusammenhang interessiert ein weiteres, besonders rätselhaftes Motiv, das auf den Begriff des Mythos zurückführt: „In dem Traum, in dem jeder Epoche die ihr folgende in Bildern vor Augen tritt, erscheint die letztere vermählt mit Elementen der Urgeschichte, das heißt der klassenlosen Gesellschaft." ${ }^{44}$ Benjamin hat hierfür einige gute Beispiele, namentlich das Schlaraffenland, erläutert jedoch nicht nachvollziehbar, wie die „Erfahrungen“ einer solchen Urgeschichte, die „,im Unbewussten des Kollektivs ihr Depot haben“"45 sollen, in die Gegenwart gelangt sind. Die bestmögliche Erläuterung könnte daher sein, dass Benjamin mit diesem Verweis einen Gegenmythos aufzubauen versucht.

Rekonstruiert man seine Bezugnahmen auf Zweideutigkeit unter diesem Aspekt, ergibt sich eine Argumentation, die mit seinen Aufzeichnungen zumindest kompatibel, auch aus seiner Situation zu begreifen und vielleicht sogar in andere geschichtlich-politische Kontexte übersetzbar ist. Zweideutig ist grundsätzlich alles Symbolische, das so oder auch anders gelesen oder gesehen werden kann. In Handlungssituationen können alle wahrgenommenen Zeichen, die auf verschiedene Fortgänge (Annäherung oder Abwendung, Entspannung oder Eskalation, Sieg oder Niederlage) zugleich hinweisen, als zweideutig gelten. Überträgt man dies auf geschichtliche Situationen und ihre symbolische Verdichtung, steht das Zweideutige für die Kopräsenz alternativer Zukunftsszenarien. Eine Rekonstruktion vergangener Zukünfte, die sich aus dieser Offenheit speist, droht nun allerdings in die alte, oben erörterte mythische Zweideutigkeit abzugleiten, wenn sie die Vergangenheit nicht nur kausal nachzuvollziehen versucht, sondern ihr zugleich Anweisungen entnimmt, wie man die eigene Lage zu verstehen hat. Die Beispiele reichen von Erzählungen nationaler Erneuerung bis zu der in Benjamins Geschichtsthesen wichtigen Idee, dass Niederlagen in früheren Befreiungskämpfen zu rächen sind. Benjamin vermeidet es zumeist, in dieser Weise zu mythisieren, indem er keine fertige Erzählung anbietet, sondern nur betont deutungsoffene Bilder. Doch eine Vergangenheit, von

43. Ibid., S. $46 f$.

44. Ibid., S. 47.

45. Ibid. 
der man sich gar nicht in praktisch entscheidender Hinsicht gemeint sieht, ist keine geschichtliche. Und ganz ohne Motive wie die klassenlose Gesellschaft, die am Ursprung stand und zu der es weiterhin oder erneut hinzieht, sieht die Konzentration ökonomischer Macht und staatlicher Herrschaft trotz aller „Unentschiedenheit“ im „Zwischenstadium“46 ziemlich alternativlos aus.

Die unausgesprochene Frage der Exilanten könnte daher sein, inwiefern es Geschichtsbewusstsein ohne Mythos geben kann. Das Gemeinsame ihrer Antworten liegt, wie ich abschließend zeigen will, in anti-identitären und anti-konservativen Umgestaltungen des Mythischen.

\section{Deutsche Menschen: Arbeit am Mythos im Exil}

Dass für Benjamins wiefürAdornosArbeitam MythosihrejeweiligenExilerfahrungen, genauer ihre Flucht vor den Nationalsozialisten, prägend waren, liegt auf der Hand. Benjamin arbeitet auch direkt gegen völkische Mythenbildung an, am deutlichsten in der 1936 unter dem Pseudonym Detlef Holz herausgegebenen und kommentierten Briefsammlung Deutsche Menschen. Bereits das Motto des Buchs lässt die Absicht erkennen, eine Art deutsches Pathos für oppositionelle Inhalte zu entwickeln: „Von Ehre ohne Ruhm / Von Größe ohne Glanz / Von Würde ohne Sold“" ${ }^{47}$ Und schon der Kommentar zum zeitlich ersten, von Lichtenberg stammenden Brief zeigt, wie sich diese Absicht in einer fein unterscheidenden, ebenfalls nah am Klischee deutscher Echtheit operierenden Urteilshaltung verwirklichen kann:

In einer Umwelt, die in ihren Tagesmoden vom Geist der Empfindsamkeit, in ihrer Dichtung vom genialischen Wesen erfüllt war, prägen unbeugsame Prosaisten, Lessing und Lichtenberg an der Spitze, preußischen Geist reiner und menschlicher aus als das fredericianische Militär. ${ }^{48}$

Adorno scheint zunächst eher an der Erfahrung des Exils selbst zu arbeiten; schließlich kann die Odyssee als der Exilmythos schlechthin gelten. In der Rekonstruktion zeigte sich jedoch, dass ein Hauptbezugspunkt seiner Deutungen die gelehrtendeutsche Gräkophilie ist, die durch viele Kanäle in die nationalsozialistische Philosophie und Geisteswissenschaft eingemündet war. In diesem Feld wird auch besonders greifbar, was sich im Verhältnis von Geschichte und Mythos ändert, wenn die reflektierte Exilerfahrung eingreift.

Als Kontrastfolie bietet sich Heidegger an, der bereits in Sein und Zeit darlegt, dass wir nur insofern geschichtliche Wesen sind und mit der Tradition brechen können, als wir vergangene Möglichkeiten aktualisieren - und der dies bereits in seinem Hauptwerk nur mit Begriffen wie „Schicksal“ und „Volk“ zu denken vermag. In Heideggers Texten der 1930er Jahre wird dann ein stärkerer Mythosbezug wichtig, den in allgemeiner und unverdächtiger Form am besten Hans Blumenberg ausgeführt

46. Ibid., S. 54.

47. Benjamin, Deutsche Menschen. Eine Folge von Briefen, in Gesammelte Schriften, Bd. IV, S. 149253, hier 150 .

48. Ibid., S. 153. 
hat: Eine Welt zu bewohnen heißt über Geschichten bzw. Deutungsmuster verfügen, mit denen wir unsere Lebensvollzüge und unsere Umgebung auslegen.

Welt zu haben ist immer das Resultat einer Kunst, auch wenn sie in keinem Sinne ein „Gesamtkunstwerk“ sein kann. Davon [...] ist unter dem Titel „Arbeit am Mythos“ etwas zu beschreiben. [...] Zur Behauptung vor der übermächtigen Wirklichkeit über Jahrtausende hinweg werden sich Geschichten, denen nicht von der Wirklichkeit widersprochen werden konnte, durchgesetzt haben. ${ }^{49}$

Beim Stichwort der ,übermächtigen Wirklichkeit“ kann man auch an Adorno denken, doch anders als bei diesem ist kein Prozess zwischen Mythos und Aufklärung postuliert. Vielmehr erhält die phänomenologische Einsicht, dass wir uns immer schon in einer ausgelegten Welt bewegen, hier eine äußerst mythosaffine Fassung. Heidegger fasst die Ausgelegtheit der Welt in den 1930er Jahren so eng, dass sich sein Interesse phasenweise vom erzählten Prozess zur sinnlichen Gegenwart maßgeblicher Kunstwerke verschiebt. Das Resultat ist ein homogener (wenngleich immer bedrohter) kultureller Kosmos, der auch die Geschichte umschließt und in dessen Mittelpunkt etwa ein Tempel steht:

Das Tempelwerk fügt erst und sammelt zugleich die Einheit jener Bahnen und Bezüge um sich, in denen Geburt und Tod, Unheil und Segen, Sieg und Schmach, Ausharren und Verfall - dem Menschenwesen die Gestalt seines Geschickes gewinnen. Die waltende Weite dieser Bezüge ist die Welt eines geschichtlichen Volkes. ${ }^{50}$

Diese Auffassung von Geschichte als Volksgeschick ist, selbst wenn Heidegger nach seinem ersten Vorstürmen Schwierigkeiten im neuen Regime hatte, voll mit dem mythisierenden Selbstverständnis des Nationalsozialismus vereinbar. Gegen eine solche Reaktualisierung des Mythos entwickeln Benjamin, Horkheimer und Adorno ihre Verschränkungen von Mythos und Fortschrittsgeschichte bzw. die Übersetzung mythischer Zweideutigkeit in unentschiedene geschichtliche Situationen. Sie fragen nach einer Welt und einer sinnlichen Wirklichkeit, die nicht vorrangig durch Volks-, Kriegs- und Schicksalsmythen erschlossen ist, sondern durch kommerzielle Ästhetik, Ideologien und Klassenkämpfe, Triebdynamik und die Umwege, die sich unterbundene Wünsche suchen. Die Grundlinien sinnlicher Weltverhältnisse und geschichtlicher Selbsterzählungen, die Heidegger weiterhin bündeln und sammeln will, werden damit insgesamt ins Exil gezogen.

49. Hans Blumenberg, Arbeit am Mythos, Frankfurt a. M. 1979, S. 13.

50. Martin Heidegger, „Der Ursprung des Kunstwerkes“, in ders. Holzwege, 7. Aufl., Frankfurt a. M. 1994, Vittorio Klostermann, S. 1-74, hier 28. 
